\title{
The Role of the SaToTM Pan Toilet Technologies in Advancing Progress in the Water, Sanitation and Hygiene (WASH) Sector
}

\author{
Jasmine Burton ${ }^{1}$, James B. Tidwell², Jenala Chipungu ${ }^{3}$, \\ Robert Aunger ${ }^{4}$
}

${ }^{1}$ London School of Hygiene and Tropical Medicine, Public Health, London, UK

${ }^{2}$ London School of Hygiene and Tropical Medicine, Behavioural Science, London ,UK

${ }^{3}$ Centre for Infectious Disease Research in Zambia, Social Behavioural Research, Lusaka, Zambia

${ }^{4}$ London School of Hygiene and Tropical Medicine, Evolutionary Public Health, London , UK

https://doi.org/10.38126/ISPG160203

Corresponding author: Jasmine Burton; jasminekburton@gmail.com

Keywords: WASH; WASH infrastructure; sanitation; toilets; toilet

Executive Summary: The United Nation's (UN) 6th Sustainable Development Goal seeks to enable the availability and sustainable management of water and sanitation for all by 2030 (UN Sustainable Development Goals Platform). Lusaka, Zambia has thirteen peri-urban areas (PUAs), all with poor sanitation conditions (WHO / UNICEF). The SaTo ${ }^{\text {TM }}$ brand, created by the LIXIL Corporation, offers a suite of frontier sanitation technologies including various safe and affordable toilet products for use in lower-and middle-income countries (LMICs) in Africa and Asia. This study examines the potential market demand and environment for the SaTo ${ }^{\text {TM }}$ Pan Sit and Squat toilets in the Bauleni PUA. In Lusaka, landlords (LLs) hold the decision-making power regarding household-based infrastructure; therefore, this study measures LL interest in the SaTo ${ }^{\text {TM }}$ Pan Toilet technologies for the LL's personal and familial use as well as for use by their tenants. 30 semi-structured interviews with conveniently sampled, adult LL residents of Bauleni were conducted to capture their demographic, sanitation, and toilet history status as well as their SaTo ${ }^{\mathrm{TM}}$ Pan product interaction. Quantitative data was analyzed in Stata; qualitative data was analyzed via thematic content analysis. Results indicate that there is an overarching positive preference toward the $\mathrm{SaTo}^{\mathrm{TM}}$ Pan Sit toilet compared to all other presented sanitation technology alternatives. Conducting a SaTo ${ }^{\text {TM }}$ Pan Sit toilet user trial and social marketing campaign pilot could potentially aid in efforts to effectively and affordably decrease the incidence of water, sanitation and hygiene (WASH)-related disease transmission in Zambia.

\section{Introduction}

4.5 billion people lack safely managed sanitation (WHO). In lower- and middle-income countries (LMICs), people often share dry pit latrines, or basic holes in the ground, that lead to health problems. Lacking improved water, sanitation and hygiene (WASH) infrastructure leads to the spread of WASHrelated diseases that "remain among the major causes of death in children under five" (UN Sustainable Development Goals Platform 2019). In 2015, approximately " $53 \%$ of the urban population in LMIC still [relied] on on-site, unimproved sanitation, such as unimproved pit latrine and open defecation" (Roma and Biran 2016). As a result of this, the World Bank reports that "poor sanitation results in a 1.3 per cent loss to Zambia's national Gross Domestic Product annually" ("Water, Sanitation and Hygiene" 2020).

Therefore, safely managing fecal waste has become a multisector priority. However, the provision of safe and sustainable sanitation products and services in 
peri-urban areas (PUAs) has proven to be incredibly complex (Blackett and Hawkins 2016). This complexity is a result of a myriad of restrictions including hardware constraints (such as toilet technologies) and societal constraints (such as behavior change, private sector support, policy support, and legal enforcement). Numerous frontier sanitation technologies have since been developed to improve the user experience for both the latrine users and for latrine emptying service providers around the world. However, there are very few market-ready solutions that have been proven at scale (Roma and Biran 2016). There is a sense of global urgency around the scale-up and accessibility of frontier sanitation technologies.

\section{i. Background of the sanitation landscape in Lusaka, Zambia}

Zambia's capital, Lusaka, has over two million people (Roma and Biran 2016; Central Statistical Office Zambia 2014). Rapid urbanization resulted in the development of thirteen PUAs across the city with little formal infrastructural planning (Water and Sanitation for the Urban Poor 2015; World Bank 2015). Approximately 56\% of Zambia's overall population and $95 \%$ of the PUA population uses unimproved sanitation facilities (Roma and Biran 2016; WHO/UNICEF 2019).

In 2015, the World Bank approved $\$ 65$ million to be utilized for the Lusaka Sanitation Project (LSP) to "increase access to sanitation services in selected areas of Lusaka and strengthen the Lusaka Water and Sewerage Company (LWSC) capacity to manage sanitation services" (World Bank 2015, Lusaka Sanitation Project 2016). The project's stated objectives are to expand waterborne sewerage systems to two PUAs, to develop a more standardized response to on-site fecal sludge management (FSM) challenges for non-sewered PUAs, and to provide technical assistance to LWSC ("Re-Inventing the Toilet: a New Era of Safe Non-Sewered Sanitation", n.d.). In September 2017, the project sought to target 216,000 people who were to receive 12,000 toilets, 10 decentralized wastewater treatment systems and 4 FSM systems sanitation facilities (Chipungu, J. 2016; Lusaka Sanitation Landscape Report; World Bank). The LSP is ongoing and is projected to close in December 2020.

\section{ii. SaTo ${ }^{\text {TM }}$ toilets in Lusaka}

This study selected two of the SaToTM Pan Toilet technologies for assessment of general perceptions because of their market readiness, widespread availability, and public health relevance as an improved pour-flush sanitation option by the World Health Organization (WHO) and United Children's Fund (UNICEF)'s Joint Monitoring Program for Water Supply and Sanitation standards (SSWM).

\section{iii. SaTo TM Pan Toilet products}

The SaTo ${ }^{\mathrm{TM}}$ brand, created by the LIXIL Corporation, is a frontier technology that "offers safe, affordable, and durable solutions for a better and more hygienic toilet experience" throughout the LMICs in Africa and Asia (SATO, American Standard). The original SaTo ${ }^{\mathrm{TM}}$ Pan is a "simple plastic pan with a counterweighted, self-closing door that seals pit latrines to prevent the passage of flying insects and thus spread of disease" (SATO) (Figure 1). It operates as a pit latrine upgrade and pour flush toilet. The wide flange $\mathrm{SaTo}^{\mathrm{TM}} \mathrm{Pan}$ Toilet is an oblong squat pan that includes wings that the user steps on while squatting. The SaTo ${ }^{\mathrm{TM}}$ Pan sit toilet is raised and allows the user to sit on the pan. It is also intended to be easily installed using mud and wood. They both act as pour flush toilets where water must be poured inside after use (Figure 1) (USDP).

\section{Various SaTo ${ }^{T M}$ Pan Toilet Products}



Figure 1: Various $\mathrm{SaTo}^{\mathrm{TM}}$ Pan Toilet Products 
LIXIL currently manufactures toilet technologies in Bangladesh, Uganda, and Kenya, and reports that over 810,000 of the first SaTo ${ }^{\mathrm{TM}}$ Pan Toilets have been distributed and installed in Bangladesh, Uganda, Haiti, Malawi, Nepal, Nigeria and the Philippines (LIXIL). LIXIL also states that "by working with an established local manufacturer, the $\$ 1.85 \mathrm{SaTo}^{\mathrm{TM}} \mathrm{Pan}$ cost made them attainable for a population surviving on less than \$1.00 per day" in Bangladesh (Research and Development Magazine 2016). This SaTo'M Pan study in Zambia was not been endorsed or sponsored by LIXIL.

\section{iv. Study rationale}

As of 2019, there is a global sanitation product mapping that exists in unpublished data from the London School of Hygiene and Tropical Medicine (LSHTM). There are over 40 innovative toilet options operating beyond pilot phase and prototype phase in different regions around the world (Roma and Biran 2016). Each of these sanitation solutions have different functionalities based on their target users and the waste management services available. In the southern Africa region, some frontier toilet technologies that currently exist include the Arumloo, EnviroSan, SavvyLoo, WeeStand and SafiChoo (Figure 2). These models are largely being tested in various pilots and or are scaling primarily in South Africa specifically. There are therefore few existing, commercially available options on the Lusaka sanitation market beyond ceramic toilet bowls and squat pans, which are expensive and largely inaccessible to the PUA populations.

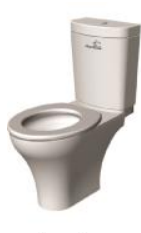

Arumloo

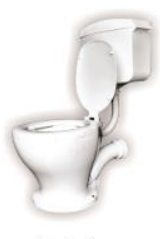

EnviroSan

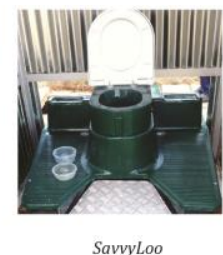

SavvyLoo



WeeStand

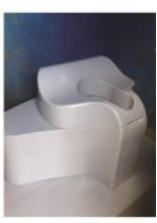

SafiChoo
Figure 2: Various Toilet Innovations in the

Southern Africa Region (images available on toilet company's websites)

The overall objective of this study is to determine the potential market demand for the frontier SaTo ${ }^{\mathrm{TM}} \mathrm{Pan}$ Toilet technologies relative to existing toilet options on the market and potential future toilet innovations in the Bauleni PUA and Lusaka at large. Ultimately this study seeks to contribute to the scope and impact of other large-scale Lusaka sanitation projects working to quell the negative health and economic consequences of poor WASH infrastructure.

\section{Methods}

\section{i. Study design}

A series of semi-structured interviews were conducted with adult landlord (LL) residents of the Bauleni PUA to determine their personal interests and perceptions of the SaTo ${ }^{\mathrm{TM}} \mathrm{Pan}$ Squat toilet versus the $\mathrm{SaTo}^{\mathrm{TM}}$ Pan Sit toilet for their personal/familial use compared to for use by their tenants. The focus is on LLs because they have the ultimate decisionmaking and purchasing power when it comes to household infrastructural renovations such as changing a plot's toilet (SHARE consortium 2016).

\section{ii. Sampling criteria}

30 purposively and conveniently sampled adult LLs who reside in the Bauleni PUA were interviewed based on who was available, met the inclusion criteria, and consented to participation. With support of the San-Dem Research Assistant from LSHTM, an inclusion criterion was determined based on previously collected demographic/geographic information (SHARE Consortium 2016). Within the 6 zones of the PUA, a diverse cross-section of LLs were identified who varied based on sex (male vs. female), age (young vs. old), socioeconomic status (high vs. low), and toilet status (shared with tenants vs. separate from tenants) in order to develop baseline understanding about the market interest and social marketing opportunities for these toilet technologies.

\section{iii. Interview guide components}

The interview guide was comprised of question types drawn from the formative research data collection tools utilized for the other concurrent WASH projects conducted by other research teams from the LSHTM and the Centre for Infectious Disease Research in Zambia (CIDRZ), the Behavior-Centered Design (BCD) Toolkit, and questions previously used from the 2016 SaTo $^{\text {TM }}$ Pan trial pilot in Uganda and Kenya (LIXIL 2016; Lusaka Water and Sewerage Company 2016). Basic demographic questions such as "How many people live on your plot?" and "Who provides the main income for [your] home?" were built upon from the data collection tools utilized by San-Dem (Tidwell 2017). The sanitation status portion of the 
guide assessed the reported WASH status of each interviewed LL's plot. Toilet observation assessed the current toilet status of the LL's household in relation to the plot. LLs were shown the SaTo ${ }^{\text {TM }}$ Pan Squat and Sit toilet technologies and were asked to observe and describe them and then to choose between the $\mathrm{SaTo}^{\mathrm{TM}}$ Pan Squat toilet, the SaTo ${ }^{\mathrm{TM}}$ Pan Sit toilet, and their current toilet for their preferred choice for themselves to use, their guests to use, and their children to use. LLs were given 5 printed images of different toilet technologies and asked to rank them: a traditional ceramic sit toilet that is currently available on the market, a ceramic squat toilet that is currently available on the market, the SaTo ${ }^{\mathrm{TM}}$ Pan Sit toilet, the SaTo ${ }^{\mathrm{TM}}$ Pan Squat toilet, and a white plastic sit toilet that is not currently on the market but is similar to the EnviroSan (Minh et al. 2013). This activity captured LL perception of an array of sanitation technologies to determine where the $\mathrm{SaTo}^{\mathrm{TM}}$ Pan Toilets technologies fit in comparison to other competitive product options. LLs were asked to indicate their own sanitation responsibilities in response to provided statements using a sheet with three smiley faces indicating "agreement," "inbetween," and "disagreement."

\section{iv. Data collection and analysis}

Interview analysis methods included developing abridged English transcripts and locating themes that were in line with the study objectives. Demographic, sanitation and toilet history data were tabulated and converted into percentages in Stata version 14 to determine the proportion of LLs with each characteristic. The data from the BCD inspiredtechnology showcase and interaction was analyzed via the qualitative research methodology of thematic content analysis (Aunger and Curtis 2015; Anderson 2007). Data collected from the Toilet Preference and Ranking Activity portions of the interviews were tabulated in Stata version 14 and the mode was captured for the 3 questions related to the $\mathrm{SaTo}^{\mathrm{TM}} \mathrm{Pan}$ Toilet technologies and their current toilet for themselves to use, their guests to use and their children to use (StataCorp 2015). The mode for each segment of the ranking activity was captured to determine which of the five toilets was preferred and disdained the most frequently. For the 'LL Perceptions of Tenants Activity', there were fourteen questions asked that were subsequently answered with the LL's referring to the smiley face-Likert scale.
These data were tabulated to determine the proportion of LLs that agreed, disagreed or were neutral in response to tenant-related statements in order to best determine the LLs existing perceptions of their own compared to their tenants' values for and responsibility of a high-quality toilet.

\section{Results}

\section{i. Demographic, sanitation status, and toilet history}

15 of the surveyed LLs relied on water from their neighbors with 14 of LLs having water on their plots and 1 having to rely on a community well source. Nearly half of LLs use dry pits with a few of them having had a dry pit latrine that collapsed due to the rainy season or to faulty construction alone. A quarter of LLs use flushing toilets connected to a septic tank and half of the LLs reported having only one toilet on their plot. Nearly a third of LLs did not know how much it cost them to construct their toilets while a quarter of them reported that they paid between 1500-3000 ZMK where 10 ZMK 1 USD (Coin Mill Currency Conversion 2017). Over a third of the LLs stated that they had between five-ten people using their latrine while a quarter of the LLs had sixteentwenty people using their latrine each day.

\section{ii. Technology showcase and interaction}

Through thematic content analysis, results were categorized, coded, and distilled into seventeen qualitative themes as seen in Figure 3 (Aunger and Curtis 2015; Anderson 2007). The SaToTM Pan Squat toilet was largely spoken about negatively or thought of as a child's toilet, bath, or urinal, while the SaTo ${ }^{\mathrm{TM}}$ Pan Sit toilet was viewed more favorably.

The qualitative theme of improved 'user experience' had a high frequency and over 32 predominant quotes across all of the transcripts with numerous references to the SaTo ${ }^{\mathrm{TM}}$ Pan Sit toilet; however, for the product showcase portion of the interviews, none of the interviewed LLs actually sat on the toilet technology despite being invited to do so. Many LLs wanted to purchase the products immediately, particularly the SaTo ${ }^{\mathrm{TM}}$ Pan Sit toilet, despite the fact that there were prototype breakages held together by duct tape. The innovative counterweight seal of the $\mathrm{SaTo}^{\mathrm{TM}}$ Pan Toilet technologies proved to be of interest but was not necessarily intuitively understood to be a lid by all interviewees. There was 


\begin{tabular}{|c|c|}
\hline Theme & Examples \\
\hline $\begin{array}{l}\text { 1. Cleanliness } \\
\text { (The desire to live a hygienic, disease free } \\
\text { life) }\end{array}$ & "[The SaTo ${ }^{\mathrm{TM}} \mathrm{Pan}$ Sit] is a clean toilet." \\
\hline $\begin{array}{l}\text { 2. Quality } \\
\text { (Both the product and building integrity } \\
\text { or durability) }\end{array}$ & "The cheap is expensive." \\
\hline $\begin{array}{l}\text { 3. Convenience } \\
\text { (Bundling product and installation } \\
\text { costs/materials to improve the customer } \\
\text { experience.) }\end{array}$ & $\begin{array}{l}\text { "We would pay } 300 \mathrm{~K} \text { for the } \mathrm{SaTo}^{\mathrm{TM}} \text { Pan squat toilet package for } \\
\text { ourselves because everything is included." }\end{array}$ \\
\hline $\begin{array}{l}\text { 4. User Experience } \\
\text { (The improved toilet usability for all } \\
\text { people) }\end{array}$ & $\begin{array}{l}\text { "Because the } \mathrm{SaTo}^{\mathrm{TM}} \text { Pan Sit is more comfortable because I have } \\
\text { problems with my legs, so usually when I use the squat toilet, I am usually } \\
\text { pained." ;"If you are tired you can sit easily on the SaTo }{ }^{\mathrm{TM}} \text { Pan Sit" }\end{array}$ \\
\hline $\begin{array}{l}\text { 5. LL and Tenant similarity } \\
\text { (The collectivist, supportive and familial } \\
\text { community mentality) }\end{array}$ & $\begin{array}{l}\text { "I don't regard tenants to have lower class things. I put more into it so } \\
\text { that they can take care of it" }\end{array}$ \\
\hline $\begin{array}{l}\text { 6. LL and Tenant Difference (The } \\
\text { sentiment that LLs and tenants are } \\
\text { different types of people.) }\end{array}$ & $\begin{array}{l}\text { "I prefer the } \mathrm{SaTo}^{\mathrm{TM}} \text { Pan sit if it was it was occupied by just me and my } \\
\text { family. I would use the SaTo }{ }^{\mathrm{TM}} \text { Squat if I was sharing with tenants." }\end{array}$ \\
\hline 7. Variance of Handyman costs & $\begin{array}{l}\text { "Plumbers are dependent; I wouldn't know what price unless I hear and } \\
\text { agree with them." }\end{array}$ \\
\hline 8. Water Reliability & $\begin{array}{l}\text { "We had a tap on our plot, but water doesn't come out." [expressing } \\
\text { concern about needing water for the SaTo }{ }^{\mathrm{TM}} \text { Pan technologies] }\end{array}$ \\
\hline 9. Free & "I don't have money I can only have it if it is for free." \\
\hline $\begin{array}{l}\text { 10. Space } \\
\text { (Related to running out of or making } \\
\text { more space for a latrine.) }\end{array}$ & $\begin{array}{l}\text { "I saw that the other was getting full so I decided to build another [by } \\
\text { digging a hole right next to it]." }\end{array}$ \\
\hline $\begin{array}{l}\text { 11.. Value of Toilets } \\
\text { (How much the LLs and their tenants } \\
\text { value toilets) }\end{array}$ & "If the toilet is good the price of the house increases." \\
\hline 12. Toilet lid as a sign of status & $\begin{array}{l}\text { "We like the SaTo }{ }^{\mathrm{TM}} \text { Pan sit toilet. But it needs a cover." [representing the } \\
\text { need for some education around the product's features in this } \\
\text { community] }\end{array}$ \\
\hline $\begin{array}{l}\text { 13. Responsibility } \\
\text { (The perceived responsibilities of } \\
\text { maintaining a toilet.) }\end{array}$ & $\begin{array}{l}\text { "The [tenants] don't help- it is the responsibility of the landlord to fix the } \\
\text { toilet." }\end{array}$ \\
\hline $\begin{array}{l}\text { 14. Laziness } \\
\text { (Not cleaning or installing the toilet as a } \\
\text { LL as a sign of laziness.) }\end{array}$ & $\begin{array}{l}\text { "Not installing the SaTo"TM Pan Squat toilet represents laziness. Look at it. } \\
\text { This is a do it yourself." }\end{array}$ \\
\hline $\begin{array}{l}\text { 15. SaTo }{ }^{\mathrm{TM}} \text { Pan Squat Toilet } \\
\text { Impressions }\end{array}$ & $\begin{array}{l}\text { "I don't like the way that the SaTo }{ }^{\mathrm{TM}} \text { Squat looks." } \\
\text { "The SaToTM Squat looks like a tub or dish to bathe a child" }\end{array}$ \\
\hline 16. SaTo ${ }^{\mathrm{TM}}$ Pan Sit Toilet Imp & $\begin{array}{l}\text { "The SaTo }{ }^{\mathrm{TM}} \text { Pan Sit is easy to install since it already has hole } \\
\text { need to make the holes bigger." }\end{array}$ \\
\hline
\end{tabular}

Figure 3 A compilation of the 17 Categorical Themes that emerged from the 30 interviews 


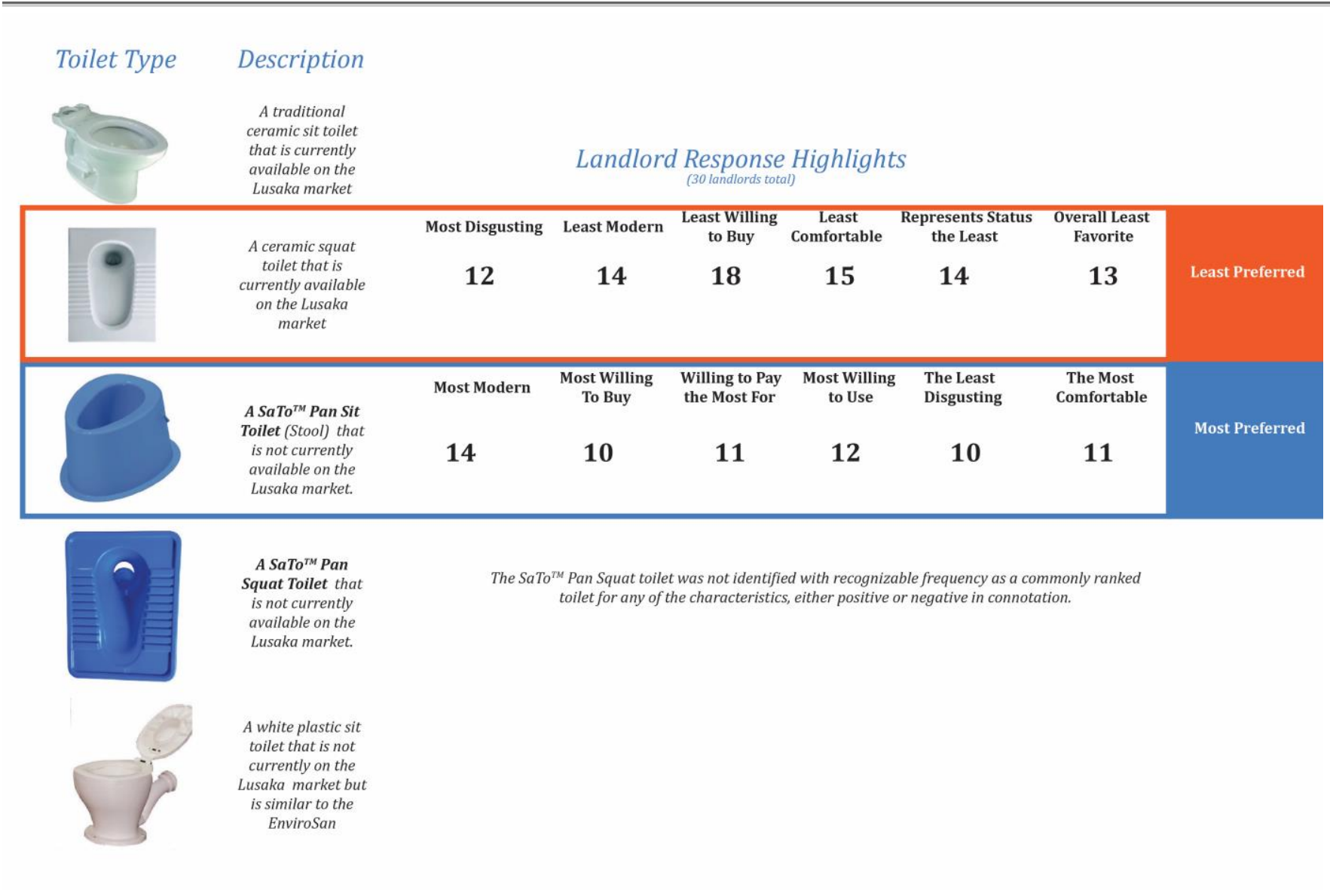

Figure 4: These are the images of the toilets that were shown to the studies interviewees. The highlighted LL responses showcase the least and most preferred options of the 5 toilets that were presented. (graph made by Jasmine Burton; photos taken by Jasmine Burton)

also an assumption that the two products fit together to make one larger toilet product rather than being two independent frontier sanitation technologies, which was evidenced by the number of people who tried to place them together or said statements like "I see that this is something that you put on top of the other toilet".

The $\mathrm{SaTo}^{\mathrm{TM}}$ Pan installation responses varied based on the LL's technical knowledge. More technically experienced respondents tended to be overly analytical and critical about the installation needs whereas laymen thought that it would be easy to install with cement. There was an overarching concern about cleanliness and hygiene. Many LLs were renovating their toilets and expressed interest in investing in something more long term and expressed that plastic was "only temporary." The materiality responses varied with positive responses such as "the rare part about it is that it is plastic. I haven't seen it with a toilet sitter" and "this plastic is [strong] and different from the plastic that we use" as well as negative responses such as "I don't like the SaTo ${ }^{\text {TM }}$ Pan, it's too plastic" and "How durable can it be since it's plastic?"

Lastly, convenience was a frequent theme attributed to these technologies and related to making the toilet purchasing/installing process more accessible.

\section{iii. Toilet preference}

Overall, the preference rankings showed 16/30 LLs found the SaTo ${ }^{\mathrm{TM}}$ Pan Sit toilet technology to be the preferred toilet for themselves, their children, and their guests to use. The only interviewees that preferred their current toilet to the SaTo ${ }^{\mathrm{TM}}$ Pans were the few that had fully flushing toilets connected to a septic tank. Some respondents reported that they would let their guests or children use the $\mathrm{SaTo}^{\mathrm{TM}} \mathrm{Pan}$ Squat toilet or their current pit latrines because 
children and guests are often messy or because the $\mathrm{SaTo}^{\mathrm{TM}}$ Pan Sit toilet technology would be special, to only be used by a nuclear family that cares for it. These responses support the perceived notion that the $\mathrm{SaTo}^{\mathrm{TM}}$ Pan Squat toilet is better for managing messier or unpredictable levels of cleanliness whereas the $\mathrm{SaTo}^{\mathrm{TM}}$ Pan Sit toilet is better for personal use with predictable cleanliness.

\section{iv. Toilet ranking}

$40 \%$ of the responses about the SaTo ${ }^{\mathrm{TM}}$ Pan Sit toilet were positively oriented as noted in Figure 4. The SaTo ${ }^{\text {TM }}$ Pan Squat toilet technology was not identified with recognizable frequency as a commonly ranked toilet for any of the characteristics, either positive or negative in connotation. The ceramic squat pan currently on the market was rated the most disgusting by nearly half of the respondents, the least modern from nearly half of the respondents, the toilet they were least willing to buy by over half of the respondents, the least comfortable by half of the respondents, the toilet that represents status the least by half of the respondents, and the overall worst toilet of the five options by half of the toilets (Figure 4). LLs most consistently stated that they would install the $\mathrm{SaTo}^{\mathrm{TM}}$ Pan Sit Toilet technology for themselves and they would be most inclined to provide the ceramic sit toilet for their tenants.

\section{v. Landlord perceptions of tenants activity}

Nearly all LLs agreed with statements (Figure 5). One of the LLs said "there should be an agreement between the landlord and tenants. It is not the job of the tenant to improve the toilet".

The findings listed in Figure 5 indicate that there is a general reported consensus that the LLs know and recognize their role in providing improved sanitation technologies for their tenants.

\section{Discussion}

\section{i. Implications}

Cleanliness was one of the major themes to arise from this study's data, specifically regarding the LL's current sanitation situation and their perceptions of the SaTo" ${ }^{\text {тм }}$ Pan Toilet technologies. The LLs who perceived the SaTo ${ }^{\mathrm{TM}}$ Pan Toilets to be unhygienic, due to either direct physical contact with the SaTo ${ }^{\mathrm{TM}}$ Pan Sit toilet or to the messy, splatter-prone perception of the SaTo ${ }^{\text {TM }}$ Pan Squat toilet, stated that they would not pay for the products for themselves, their families or their tenants regardless of price.

The provision of specific or differentiated toilet technology needs for LLs and tenants did not vary as greatly; the concept of 'shared sanitation' is generally not desirable, but because most people are focused on leading clean, hygienic lives through affordable WASH product and service offerings, cleanliness appeared to be the LL's primary concern. The cost of the toilet technology was then their secondary concern related to the $\mathrm{SaTo}^{\mathrm{TM}}$ Pan Toilet technologies. If these toilets technologies can be proven to remain clean easy to clean while also being affordable through further community-based trials, the uptake and belief in the technology in Bauleni will likely increase. However, a tension still exists for the LLs that share toilets with their tenants, particularly if they view their tenants as messy. The nuance of these findings implies that a toilet technology portfolio, inclusive of toilets with varying designs, costs, and user experiences for the end user and waste management provider, could meet the diverse sanitation needs of resource-constrained PUAs within Lusaka, while collectively working to reduce the overall burden of WASH-related diseases in Zambia.

\section{ii. Strengths and limitations}

This study's strength is that it builds on and aligns with existing WASH knowledge (both published and unpublished) in Lusaka, Sub-Saharan Africa and in the world at large. It acts as a baseline and formative market-understanding for a specific pour-flush toilet technology which could be instrumental for Lusaka as its sanitation landscape prepares for large-scale improvements and change. The sample size and timeframe of this study catalyzed a series of limitations that may impact the reliability of these findings. Relying on the interviewees' self-identifying as LLs could have also produced some selection bias. Social desirability bias was likely present as well particularly in the toilet ranking activity. Additionally, the SaTo ${ }^{\mathrm{TM}}$ Pan Sit toilet broke en route to Lusaka, which required the use of duct tape. Due to the time constraints of this study, the researchers were unable to procure another toilet sample. Triangulating this data with that of other SaTo ${ }^{\mathrm{TM}} \mathrm{Pan}$ markets studies may be beneficial in determining the extent of these limitations. 

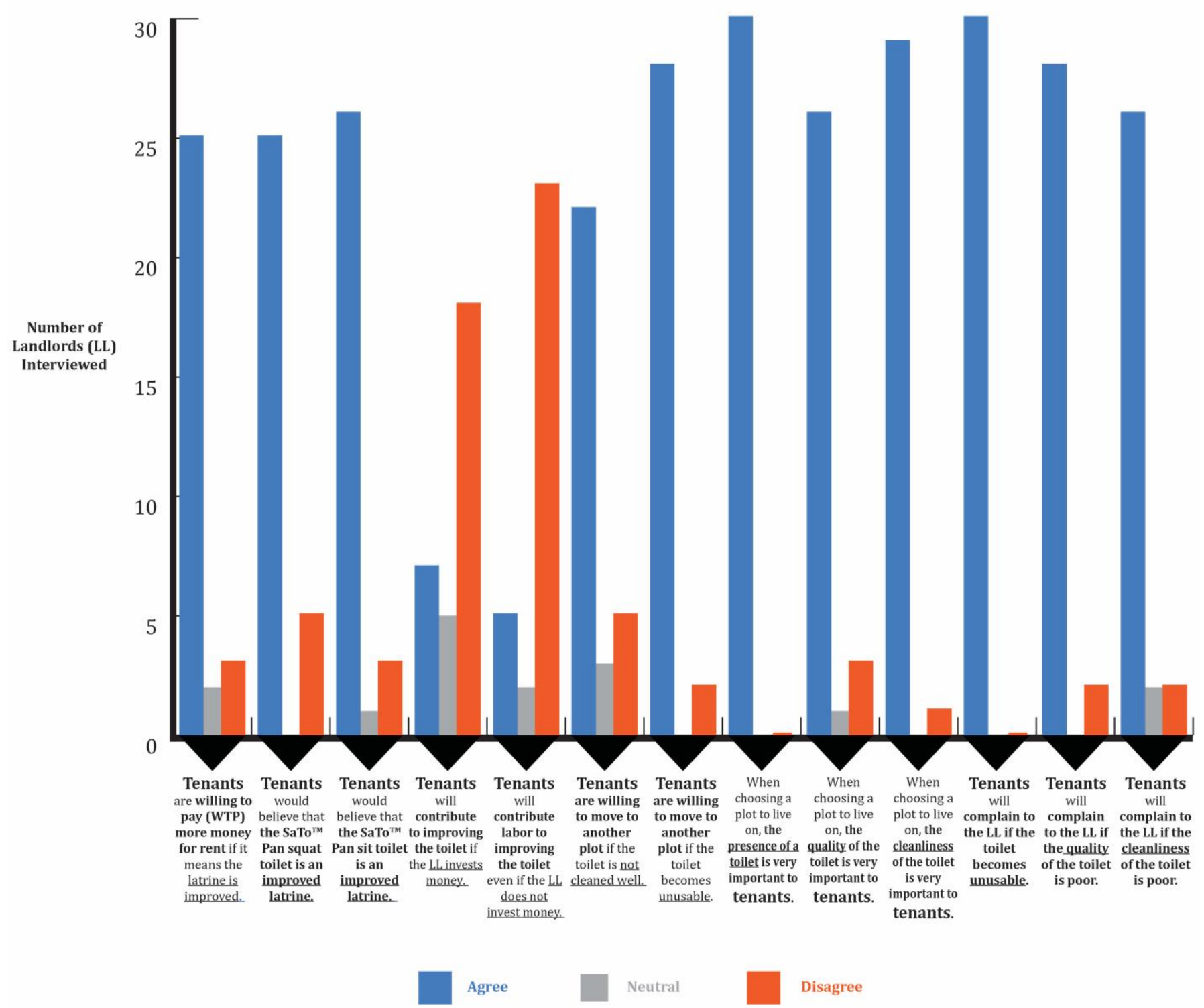

Statemets to which the
Landlords (LL)

Responded

Figure 5: This graph showcases the landlord's perceptions about their tenants

\section{Conclusions}

This study has shown that the SaTo ${ }^{\text {TM }}$ Pan Sit Toilet technology is preferred over all other presented alternatives, which represents a potential market demand for this product to fill the supply-side void for improved and affordable sanitation technologies. The LL's preference, interest and understanding of the $\mathrm{SaTo}^{\mathrm{TM}}$ Pan Squat toilet was quite negative or indifferent especially related to the themes of cleanliness, quality, and user experience.

LLs seem to collectively understand the value of sanitation, that their tenants value clean and wellmaintained toilets, and that it is largely their responsibility as LLs to invest in sanitation infrastructure for their own financial benefit (i.e. through increased rentals) and social benefit (i.e. 
wanting to protect their social capital and community standing as responsible LLs). Lusaka represents an opportunity to conduct user trials and perhaps a market viability study in an effort to capture an understanding of actual willingness-to-pay behaviors of the PUA LLs.

Much of the WASH work done in Lusaka at this time is being hallmarked as an incredible learning opportunity for other large-scale sanitation improvement projects in LMIC across Sub-Saharan Africa. With SaTo ${ }^{\mathrm{TM}}$ Pan Toilet technology trials and leanings being conducted and collected in Kenya, Uganda and Tanzania, these market research findings could potentially be of value to LIXIL for design and scaling strategy for the region. Additionally, the investment and prioritization that Zambian policymakers are making in WASH infrastructure, behavior change, and education has enabled technology innovation in this sector to grow and thrive. SDG 6's target 6.2 specifically calls for universal access to improved sanitation and hygiene while also ending open defecation by 2030 (UN Sustainable Development Goals Knowledge Platform 2019). In 2018, UN Water released a report that states that one in ten countries is currently on track to achieve universal sanitation coverage by 2030 .

\section{References}

American Standard. 2016. LIXIL Celebrates One Million SaTo ("Safe Toilet") Products in Use Globally. Retrieved March 6, 2020, from American Standard website

https://www.americanstandard-us.com/pressroom/press-releases/2016/05/31/lixilcelebrates-one-million-sato-products-in-useglobally

Anderson, R. 2007. Thematic Content Analysis (TCA) Descriptive Presentation of Qualitative Data Using Microsoft Word.

Aunger, R. and Curtis, V. A. 2015. Guide to Behaviour Centred Design. Retrieved March 6, 2020, from London School of Hygiene and Tropical Medicine website:

https://blogs.lshtm.ac.uk/envhealthgroup/files/ 2015/04/Guide-to-Behaviour-CentredDesign.compressed-2.pdf

Blackett, I., and Hawkins, P. 2016. Faecal Sludge Management Tools: Tools and Guidelines Water Sanitation Program Technical Paper. World Bank Group / Water and Sanitation Program. http://documents.worldbank.org/curated/en/46 1321468338637425/pdf/106805-REVISED.pdf
"Substantial investment will be required, particularly in rapidly growing urban areas, although solutions will vary depending on the relative importance of sewerage networks and on-site sanitation systems" (UN 2018). This means that building up and further strengthening the capacity of authorities on the local and national levels will increase in priority especially in LMICs. Lusaka and Zambia at large are no exception. It could be of interest to LSP and LWSC authorities to have a set of toilet innovation products, including technologies like the $\mathrm{SaTo}^{\mathrm{TM}}$ Pan Toilet, to develop a toolbox of toilet offerings that could be afforded and effectively used by various people in Lusaka.

According to UN Water, "achieving SDG 6 is essential for progress on all other SDGs and vice versa. Sustainable management of water and sanitation underpins wider efforts to end poverty, advance sustainable development and sustain peace and stability" (UN Water 2018). Therefore, proven and sustainable frontier technologies in the WASH sector, particularly related to sanitation, have the potential to have a profoundly positive and lasting impact as it relates to global social inclusion, health equity, gender parity, public private partnership models, and the environmental movements.
Central Statistical Office (CSO) [Zambia], Ministry of Health $(\mathrm{MOH})$ [Zambia], and ICF International. 2014. Zambia Demographic and Health Survey 2013-2014. Rockville, Maryland, USA: Central Statistical Office, Ministry of Health, and ICF International.

Chipungu, J. 2016. Lusaka Sanitation Landscape Report. (Unpublished).

Coin Mill Currency Conversion. 2017. United States Dollar (USD) and Zambian Kwacha (ZMW) Currency Exchange Rate Conversion Calculator.

LIXIL. 2016. Field Testing Protocol Uganda/Kenya/UNICEF ESAR. (Unpublished).

Lusaka Water and Sewerage Company. 2016. Lusaka Sanitation Program: Improving Sanitation in the City of Lusaka. (Brochure).

"Re-Inventing the Toilet: a New Era of Safe Non-Sewered Sanitation." n.d. International Water Association, iwa-network.org/news/re-inventing-the-toilet-anew-era-of-safe-non-sewered-sanitation/.

Research and Development. 2016. American Standard's $\mathrm{SaTo}^{\mathrm{TM}}$ : A Safe Toilet. 
Roma, E., and Biran, A. 2016. Faecal Sludge Management in Urban and Peri-Urban Areas of Low and Middle Income Countries: Bottlenecks and Viable Solutions for Lusaka, Zambia (unpublished).

SATO. Smart, fresh toilets. Retrieved March 6, 2020 from SATO website http://www.sato.lixil.com/

SHARE Consortium. 2016. San-Dem. Retrieved March 6, 2020 from SHARE website: https://www.shareresearch.org/project/sandem

SSWM. 2017. Pour-Flush Toilet. Retrieved March 6, 2020, from SSWM website: https://sswm.info/content/pour-flush-toilet

StataCorp. 2015. Stata Statistical Software: Release 14. College Station, TX: StataCorp LP.

Tidwell, B. 2017. San-Dem Dataset. (Unpublished)

UN Water. 2018. Synthesis Report on Water and Sanitation. Accessed March 6, 2020 from UN website:

https://www.unwater.org/publications/highlig hts-sdg-6-synthesis-report-2018-on-water-andsanitation-2/
UN Sustainable Development Goals Knowledge Platform. 2019. Progress of Goal 6 in 2019. Retrieved March 6, 2020, from UN website: https://sustainabledevelopment.un.org/sdg6

USDP. 2015. Improved or Unimproved Sanitation. Retrieved March 6, 2020, from USDP website: http://www.usdp.or.id/id/?p=4638

Water and Sanitation for the Urban Poor. 2015. Introducing safe FSM services in low-income urban areas: lessons from Lusaka. The Stone Family Foundation, 1-11.

"Water, Sanitation and Hygiene." Related UNICEF Sites, 29 Mar. 2020, www.unicef.org/zambia/water-sanitation-andhygiene.

WHO / UNICEF. 2015. Lack of sanitation for 2.4 billion people is undermining health improvements. Retrieved March 6, 2020, from WHO website: https://www.who.int/mediacentre/news/relea ses/2015/jmp-report/en/

World Bank. 2015. FSM conditions in Lusaka, Zambia: A Rapid Assessment. (Unpublished).

World Bank. 2015). Lusaka Sanitation Project. Retrieved March 6, 2020, from UN website: http://projects.worldbank.org/P149091/?lang =en\&tab=overview

\section{Jasmine Burton}

Jasmine Burton graduated with Highest Honors from the Georgia Institute of Technology's (GT) School of Design with a BSc in Industrial/Product Design. Prior to graduation, Burton founded Wish for WASH, LLC, a social impact startup intended to bring innovation to sanitation after her senior design team was the first allfemale team to win the GT InVenture Prize Competition (the largest undergraduate invention competition in the USA) for their invention - the SafiChoo toilet. This work was catalyzed in response to the 2.3 billion people around the world today that lack safe and hygienic toilets, which is a statistic that disproportionately hinders the livelihood of women, girls and other marginalized groups. Jasmine has since led Wish for WASH in conducting iterative toilet innovation pilots and research in Kenya, Zambia, Uganda, Ethiopia and in an Atlantabased resettled refugee community with a human-centered design and gender equity lens. She continued to pursue her passion for equitable and sustainable sanitation as a Rotary Global Grant Scholar and MSc in Public Health student at the London School of Hygiene and Tropical Medicine. With 7+ years of diverse WASH (water, sanitation and hygiene) experience in the research, communications, health and gender equity spaces within the public, private, and social enterprise sectors across 10 countries, Jasmine identifies as a social impact designer and storyteller who seeks to utilize design thinking and business acumen to accelerate universal access to improved sanitation for all because \# everybodypoops.

\section{Acknowledgements}

Jasmine Burton received London School of Hygiene and Tropical Medicine (LSHTM) technical support from the San-Dem project Research Assistant and LSHTM PHD Candidate, Dr. James B Tidwell, as well as from the TRANSFORM project Research Fellow, Dr. Katie Greenland. Dr Robert Aunger, Dr. Adam Biran, and Dr. Valerie Curtis supported in the developed of my MSc project proposal and protocol; Dr. Aunger became Jasmine's direct project supervisor and aided in the refinement of my protocol, data collection tools, and written drafts. While in Lusaka, Jasmine was provided with an office space at CIDRZ Headquarters after meeting with and receiving support from the Zambian-facing LSHTM- CIDRZ TRANSFORM Principle Investigator (PI), Dr. Roma Chilengi. Jenala Chipungu and Joyce Chilekwa, both of whom were CIDRZ Research Fellows, acted as Jasmine's main in-country points of contact for training, logistics, and technical support. Financial support for this 
fieldwork was obtained via the LSHTM Trust Fund, LSHTM MSc-based bursary, and Rotary International Global Grant scholarships. During Jasmine's time in Zambia, Dr. James B Tidwell supported her with developing her sampling strategy within Bauleni. Jenala and Joyce aided in the selection of Jasmine's two Zambian project research assistants, Herbert and Ester, whom provided her with real-time interview translations.

\section{Disclaimer}

SafiChoo is a modular toilet concept created by Wish for WASH, LLC which was founded by Jasmine Burton. 